\title{
Application of Multi-order Complementary Filtering Technique in Dynamic Attitude Measurement of Forestry Special Robots
}

\author{
Bin LIU \\ Henan Forestry Vocational College \\ Luoyang, Henan, China \\ E-mail: 87570637@qq.com
}

\author{
Yan REN* \\ Henan Forestry Vocational College \\ Luoyang, Henan, China \\ E-mail: 271804648@qq.com \\ +* Corresponding author
}

\author{
Xufang DENG \\ Henan Forestry Vocational College \\ Luoyang, Henan, China \\ E-mail:277839723@qq.com
}

\begin{abstract}
The wide application foreground of forestry special robots makes it become the hotspot of current research. The attitude angle measurement [1] of the special crawler-type robot is one of the key problems. Based on the complementary filtering algorithm, the attitude and angle measuring circuit and data processing algorithm of the special crawler robot are designed. The signal filtering and preprocessing are used to combine the two sensor data. The calculation method of the key parameters in the complementary filtering algorithm is analyzed. The method is applied to the angle measurement of the special crawler robot, and the verification test is carried out. And the experiment result shows that this experiment method is applicable to forestry robots.
\end{abstract}

Keywords-forestry special robots; multi-order complementary filtering; integral drift; attitude solving

\section{INTRODUCTION}

Attitude angle measurement is a prerequisite for the operation and control of special crawler-type robots. The accuracy and speed of the attitude angle measurement [2] will directly affect the stability and reliability of the control algorithm of the special crawler robot. With the miniaturization of the inertial measurement components and the improvement of the computing power of the microprocessors, the low-cost inertial measurement unit (IMU) is widely used in posture measurement of forestry special crawler-type robots, and high accuracy attitude measurement is realized by combining the microprocessor data processing algorithms. IMU is mainly composed of low cost MEMS gyroscope and three axis accelerometer. MEMS gyroscope has good autonomy [3], low power consumption, good mechanical and electrical performance advantages of integration. However, the MEMS gyroscope has the characteristic of temperature drift; the measurement error will be accumulated with the accumulation of time, thus affecting the measurement accuracy. The accelerometer will be affected by the vibration of the robot, aliasing the additional amount of vibration interference. So a single sensor measurement is difficult to get accurate attitude angle.
The multi-sensor signal fusion method is adopted to obtain the accurate attitude angle quantity [4].

Multi-sensor data fusion methods include neural network, wavelet analysis, Kalman filtering [5] and other attitude-solving algorithms, however, these methods can establish a stable and reliable updating equation with high order, and the computation is large, which is not suitable for the real-time calculation of low computing power system. Comparing with the above method, the complementary filtering algorithm has low requirement on the speed of the processor and is simple and reliable. Based on the complementary filtering algorithm, the attitude and angle measuring circuit and data processing algorithm of the special crawler robot are designed. The signal filtering and preprocessing are used to combine the two sensor data. The calculation method of the key parameters in the complementary filtering algorithm is analyzed. The method is applied to the angle measurement of the special crawler robot, and the verification test is carried out. The experimental data are given.

\section{COMPLEMENTARY Filtering AlgORITHM}

Gyroscopes alone or accelerometers alone do not provide effective and reliable attitude information. Although gyroscope good dynamic response and not subject to the impact of acceleration changes, there is the drawback of integral error, so it is not suitable for long time alone for attitude calculation. The accelerometer has good static response, but it is susceptible to acceleration caused by nongravity, and is not suitable for tracking dynamic angular motion [6]. Based on the characteristics of the above two sensors, the complementary filtering method is adopted to combine the advantages of the two sensors.

The first-order differential system and two sets of measurement information were considered.

$$
\dot{x}=u
$$




$$
\begin{gathered}
y_{a}=u+\mu_{a} \\
y_{b}=\omega+\mu_{b}+b
\end{gathered}
$$

In the formula, ya represents accelerometer acquisition of low-frequency information; yb represents high-frequency information collected by the gyroscope; $\mu \mathrm{a}$ and $\mu \mathrm{b}$ are the measurement noise of the accelerometer and the gyroscope respectively; b- the gyro bias. The complementary filter applied to the engineering practice is shown in Fig. 1[7].

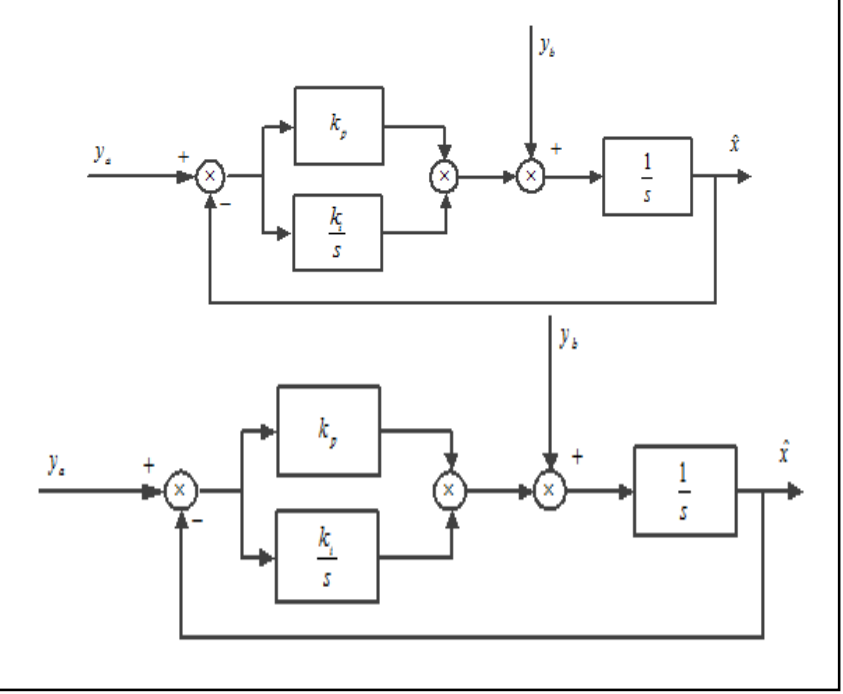

Figure 1. The complementary filter

If set $C(s)=k_{p}+\frac{k_{i}}{s}$, take $G_{L}(s)=\frac{C(s)}{s+C(s)}$ and $G_{H}(s)=\frac{s}{s+C(s)}$. Fig. 1 can be obtained:

$$
\begin{aligned}
\hat{x}(s) & =\frac{C(s)}{s+C(s)} y_{a}+\frac{s}{s+C(s)} \frac{y_{b}}{s} \\
& =x+\frac{C(s)}{s+C(s)} \mu_{a}+\frac{\mu_{b}}{s+C(s)}
\end{aligned}
$$

In the formula, $G_{L}(s)$ _Low pass filter, $G_{H}(s)$ — high pass filter.

Fig. 1 can be obtained by the dynamic differential equation of the system.

$$
\begin{aligned}
& \dot{\hat{x}}=y_{b}-\hat{b}+k_{p}\left(y_{a}-\hat{x}\right) \\
& \dot{\hat{b}}=k_{i}\left(y_{a}-\hat{x}\right)
\end{aligned}
$$

The estimated error is defined as: $\tilde{x}=x-\hat{x}, \tilde{b}=b-\hat{b}$, The function V is:

$$
V=\frac{1}{2}(x-\hat{x})^{2}+\frac{1}{2 k_{i}}(b-\hat{b})^{2}
$$

The derivative of the two ends of the function, set $y_{a} \approx u, y_{b} \approx \omega+b$, can be drawn.

$$
\begin{aligned}
\dot{V} & =(x-\hat{x})(\dot{x}-\dot{\hat{x}})+\frac{1}{k_{i}}(b-\hat{b})(\dot{b}-\dot{\hat{b}}) \\
& =\tilde{x}\left(u-(u+b)+\hat{b}-k_{p}(x-\hat{x})\right)+\frac{1}{k_{i}} \tilde{b}\left(0+k_{i}(x-\hat{x})\right) \\
& =-\tilde{x} \tilde{b}-k_{p} \tilde{x}^{2}+\tilde{x} \tilde{b} \\
& =-k_{p} \tilde{x}^{2} \leq 0
\end{aligned}
$$

According to the theorem of Lyapunov and Lassalle invariant set theorem, the system is stable.

\section{THE EXPERIMENTAL RESULTS}

In order to verify the feasibility of the above design scheme, the LR330 crawler robot developed by Henan Forestry Vocational College was used as the experimental platform. In this study, the inertial sensors of the inertial measurement unit (IMU) were selected using the ENC-03 (measuring range: \pm 300 (deg / s)) gyroscope with a sampling frequency of $1.25 \mathrm{kHz}$ and an MMA7361 accelerometer (measuring range: $\pm 1.5 \mathrm{G}$ ) [8]. The angle update frequency is $1.25 \mathrm{kHz}$. The cut-off frequency of the complementary filter is $138 \mathrm{~Hz}$. Taking the pitch angle $(\theta)$ as an example, the test is carried out. Take the robot left turn $30 \mathrm{~km} / \mathrm{h}$ as an example, Fig. 2 shows the comparison between the reference algorithm and the reference results. Table I. shows the error statistics of the algorithm. It can be seen from the table that the integral accuracy of angular velocity is higher than that of the calibrated accelerometer. The reference angle data are derived from the experimental data stored in the PC software of the commercial attitude measurement equipment. 

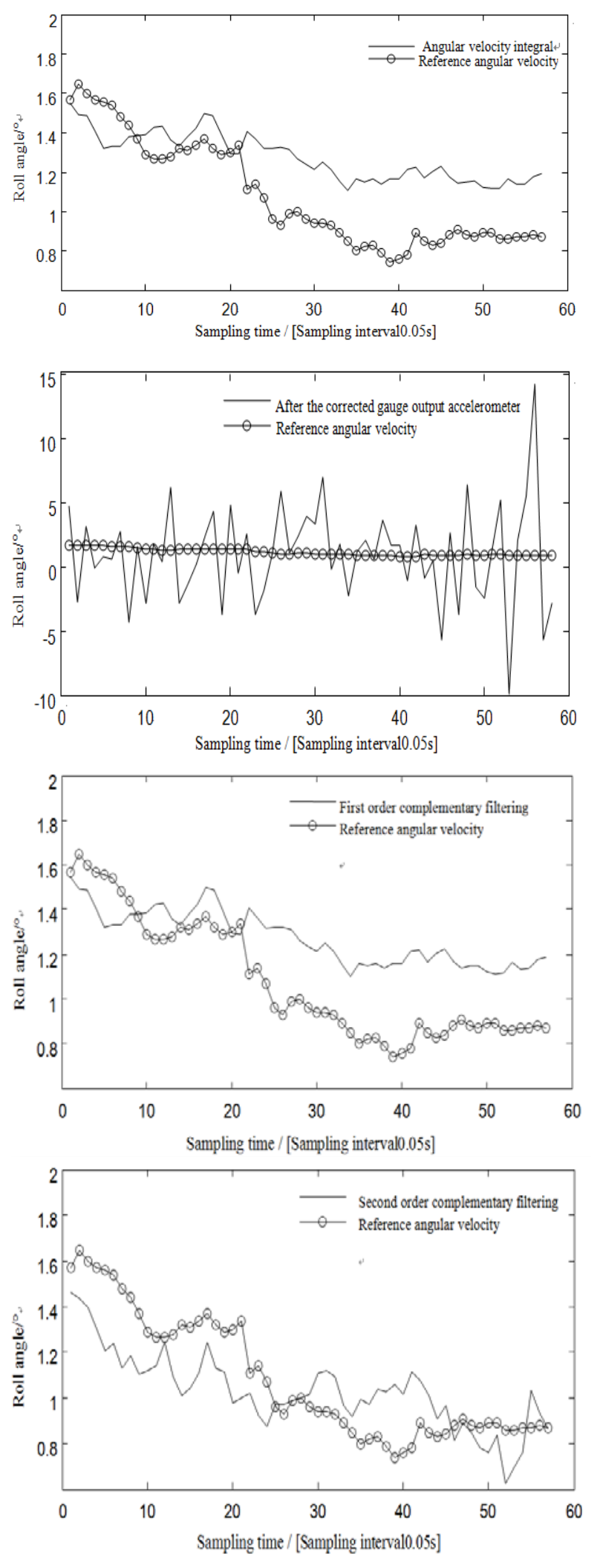

Figure 2. Comparison among diverse algorithms
TABLE I. NUMERICAL CHARACTERISTICS OF ERROR

\begin{tabular}{|c|c|c|c|c|}
\hline Algorithm & $\begin{array}{c}\Delta \gamma_{\min } \\
{ }^{\circ}\end{array}$ & $\begin{array}{c}\Delta \gamma_{\max } \\
/^{\circ}\end{array}$ & $E(\Delta \gamma)^{\circ}$ & $\begin{array}{c}D(\Delta \gamma) \\
/^{2}\end{array}$ \\
\hline $\begin{array}{c}\text { Angular } \\
\text { velocity integral }\end{array}$ & -2.889 & 0.7733 & -0.8428 & 0.5657 \\
\hline $\begin{array}{c}\text { After the } \\
\text { accelerometer is } \\
\text { calibrated }\end{array}$ & -17.0082 & 26.6352 & 0.2681 & 31.9396 \\
\hline Algorithm & $\begin{array}{c}\Delta \gamma_{\min } \\
{ }^{\circ}\end{array}$ & $\begin{array}{c}\Delta \gamma_{\max } \\
/^{\circ}\end{array}$ & $E(\Delta \gamma)^{/{ }^{\circ}}$ & $\begin{array}{c}D(\Delta \gamma) \\
\rho^{2}\end{array}$ \\
\hline $\begin{array}{c}\text { First Order } \\
\text { Complementary } \\
\text { Filtering }\end{array}$ & -2.8241 & 0.8307 & -0.8174 & 0.5490 \\
\hline $\begin{array}{c}\text { Second order } \\
\text { complementary } \\
\text { filtering }\end{array}$ & -1.6776 & 2.5994 & -0.2384 & 0.8743 \\
\hline
\end{tabular}

In TABLE I. $\Delta \gamma_{\min }$ is Angle difference minimum, $\Delta \gamma_{\max }$ is Angle difference maximum, $E(\Delta \gamma)$-Angle difference, $D(\Delta y)$-Angle difference variance.

The angular velocity data measured by the gyroscope and the angle data measured by the accelerometer, can be seen, the complementary filtering algorithm to get the angle after the elimination of the Gyroscope drift and high-frequency disturbance of the accelerometer [9], the following conclusions can be drawn:

1) The angle obtained by integrating the angular velocity obtained from the gyroscope is not correct. The angular accuracy should be improved by combining the angle value measured by the accelerometer and the angle after the gyro measured the angular velocity.

2) After the complementary filtering, the drift of the gyro is restrained obviously, which shows the effectiveness and superiority of the complementary filtering algorithm.

Using the corrected acceleration solution, the variance is larger and the value is more dispersed, the angle difference variance is larger, so this algorithm is not desirable [10]. The absolute value of the error between the first-order complementary filter and the second-order complementary filter and the reference data is the smallest and the variance is smaller and the data is more concentrated, and the variance is small and the data are concentrated.

\section{CONCLUSION}

The influence of gyroscope drift and high - frequency disturbance of the accelerometer is analyzed in the solution of attitude angle of two - wheel balance vehicle, and uses complementary filter fusion accelerometer and gyroscope signal. The complementary filter can effectively eliminate the drift of the gyroscope, suppress the high - frequency disturbance of the accelerometer, reduce the dynamic error of the output attitude angle and improve the angle measurement accuracy, which can meet the needs of attitude control of the two - wheel balance vehicle. The experimental results show that the proposed method is effective and can be applied to the attitude angle measurement system of vehicle navigation, two-wheel balance vehicle and micro-robot. 


\section{REFERENCES}

[1] SKOG I,HANDEL P,Calibration of a mems inertial measurementunit[C]. 18th IM EKO World Congress, Metrology for a Sustainable Development,Rio de Janeiro, Brazil, 2006: 10-22.

[2] DEMOZ G, ROGER C,J D POEWELL.A low-cost GPS/inertial attitude heading re ference system (AHRS) for general aviation applications[C]. Position Location and Navigation Symposium, IEEE 1998, Palm Springs, 1998: 25-32.

[3] [SERGIO D, JAVIER A, Low cost navigation system for UAV's [J]. Aerospace Science and Technology, 2005, 9(6): 504-516.

[4] Lance Sherry, Chris Brown, and Ben Montazed et al. Perfor-mance of automotive-grade MEMS sensors in low cost AH-RS for general aviation. Digital Avionics Systems Conference, Indianapolis, USA, 2003:1-5.
[5] PETER S, Integrating GPS and INS with a tightly coupled Kalman filter - using proximity information for compensating signal loss [D]. Stockholm: Royal Institute of Technology,2003:88-96

[6] Kevin Murrant, Attitude and Heading Reference System for Small Unmanned Aircraft Collision Avoidance Maneuver-s.Memoria University of Newfoundland, 2012:22-46.

[7] Christopher J. Fisher. Using an Accelerometer for Inclinat-ion Sensing [Z/OL].

[8] W.Geiger, J.Bartholomeyczik, U.Breng, et al. MEMS IM-U for AHRS applications. Position, Location and Navigatio-n Symposium, IEEE/ION, Monterey, CA (2008).

[9] Ng G W, Ng K H. Sensor management: what, why and how J. Information Fusion. 2000, 1(2):67-75.

[10] Anderson J, Hong L. Sensor resource management driven by threat projection and priorities J. Information Sciences. 2008, 178(6):2007-2021. 